\title{
Autonomous Sensory Meridian Response: Phenomenon That Promote Happiness
}

\author{
Peng Yin ${ }^{*}$, Xiao Xiao \\ ${ }^{1}$ Southwest University Faculty of Psychology, Chongqing, China \\ ${ }^{2}$ Chongqing Jiulongpo District Center for Disease Control and Prevention, Chongqing, China \\ Email: *yinpeng_psy@163.com
}

How to cite this paper: Yin, P., \& Xiao, X. (2021). Autonomous Sensory Meridian Response: Phenomenon That Promote Happiness. Psychology, 12, 321-326. https://doi.org/10.4236/psych.2021.123021

Received: October 27, 2020

Accepted: March 2, 2021

Published: March 5, 2021

Copyright (c) 2021 by author(s) and Scientific Research Publishing Inc. This work is licensed under the Creative Commons Attribution International License (CC BY 4.0).

http://creativecommons.org/licenses/by/4.0/

\section{(c) (i) Open Access}

\begin{abstract}
Autonomous Sensory Meridian Response (ASMR) describes a tingly physical response triggered by specific visual and auditory stimuli such as whispering, personal attention, crisp sounds and slow movements. Some investigations find that ASMR is associated with specific personality traits such as openness. There also exist individual differences in functional neural connectivity across individuals who do and do not experience ASMR. The similarity and high correlation between ASMR and frisson and misophonia allow us to guess the possible formation mechanism of ASMR from the perspective of those two. The high correlation with mindfulness reveals the potential for assisting psychotherapy.
\end{abstract}

\section{Keywords}

Autonomous Sensory Meridian Response (ASMR), Personality,

Positive Emotion, Trigger, Synaesthesia, Frisson, Misophonia, Mindfulness

\section{Introduction}

Autonomous Sensory Meridian Response (ASMR) is a sensory experience in which specific sensory stimuli elicit tingling sensations in the scalp and neck, often spreading to the back and other parts of the body. In addition to physiological responses, ASMR users often report positive feelings of relaxation and well-being (Barratt \& Davis, 2015).

ASMR is similar to some other sound-related sensory phenomena, such as frisson, synesthesia, and misophonia, but some psychological characteristics make it distinct from these phenomena. For example, although both frisson and ASMR can be described as "pleasant tingling", their duration is different: frisson usually occurs and spreads quickly, while ASMR has a few minutes duration 
(Roberts et al., 2020). Similarly, despite ASMR and synesthesia consistently targeting specific stimuli, individuals can choose to leave the stimulus to stop ASMR while synesthesia experience is uncontrollable (Barratt \& Davis, 2015). ASMR and misophonia are both responses to special patterns of sounds, but the emotions they bring are opposite: ASMR is often accompanied by positive emotions, while misophonia is negative (Rouw \& Erfanian, 2018).

\section{Triggers and Sensations of ASMR}

In one of the earliest research, Barratt and Davis (2015) categorized common triggers for ASMR, and where these sensations were often experienced on the body. A total of 475 participants accomplished an online questionnaire contained 7 sections which gathering information of demographics, ASMR video viewing habits, whether theses triggers are commonly present in ASMR video produce tingles, the location of tingling sensation associated with ASMR, "Why do you watch ASMR video?", flow state scale, effect on mood and chronic pain. The results showed that there are four common categories of triggers: whispering, personal attention, crisp sounds and slow movements (Barratt \& Davis, 2015). Other common preferences were soft-spoken voice particularly presented as speech sounds like " $\mathrm{s}$ ", "sh", "sk" as well as breathing, chewing, and eating sounds. As for non-vocal triggers, individuals prefer brushing, buzzing, tapping sounds, clicking or scratching, water noises and the sound of hair being combed or brushed. About sensations, individuals widely reported that typical sensations associated with ASMR was a tingling sensation which originated at the crown of head, to the back of scalp and progressed down the line of spine.

After that, they investigated the key multisensory factors contributing to the successful induction of ASMR through online media (Barratt et al., 2017). Aspects such as timing and trigger load, atmosphere, and characteristics of ASMR content, ideal spatial distance from various types of stimuli, visual characteristics, context and use of ASMR triggers, and audio preferences are explored. Lower-pitched, complex sounds were found to be especially effective triggers, as were slow-paced, detail-focused videos. Conversely, background music inhibited the sensation for many respondents. However, it is worth noting that these features, which are relatively more people selected, do not have a significant advantage. Different subjects have very different self-reports of sensitive triggers, physical responses and psychological outcomes.

\section{Individual Difference in ASMR}

These differences suggest the existence of individual differences in ASMR susceptibility and consequences. Fredborg, Clark and Smith (2018) surveyed individuals regarding five major personality domains and their ASMR experiences: 290 participants with ASMR and 290 matched controls completed the Big Personality Inventory (BFI), the former also completed a questionnaire related to their ASMR experience. Their results indicated that ASMR experiencers have a 
significantly higher score on neuroticism and openness to experience, but lower on conscientiousness, extraversion, and agreeableness. In addition, they identified five factors that could independently predict viewers' perceived intensity ratings: watching, touching, repetitive sounds, simulations and mouth sounds. Similar research was conducted by (McErlean \& Banissy, 2017). They sent the BFI and the Inter-Personal Reactivity Index (IRI) to individuals who reported experience with ASMR and matched control participants. The results showed that ASMR self-reporters score higher on openness to experience and lower on conscientiousness measures of BFI. They also showed higher scores on Empathy Concern and Fantasy subscale of IRI.

In addition to personality characteristics, there exist individual differences in functional neural connectivity across individuals who do and do not experience ASMR. Smith et al. (2017) collected the fMRI data of 22 individuals (11 in the ASMR group and 11 in the control group). The results showed that the functional connectivity of the default mode networks (DMN) in ASMR participants was significantly lower than that in the control group. The DMN of ASMR individuals also showed increased connectivity between the occipital, frontal and temporal cortical regions, indicating that ASMR is associated with a mixture of multiple resting state networks. In this study, the method used to screen the participants was to ask them to confirm the trigger list. The answer was not an instant confirmation and was easily misled by the participants' memory. In later research, they improved this method of screening participants: let the participants watch ASMR videos on-site, and confirm whether they belong to the ASMR group or the control group based on the real-time response. And this research has reached similar conclusions as before (Smith et al., 2019).

In addition to the comparison of neural network activities between ASMR experiencers and non-sensed individuals, researchers have further explored the differences in the brain activity of ASMR experiencers who are sensitive to different triggers (Smith et al., 2020). They recruited 15 people who had ASMR experience, asked them to confirm the trigger list, selected the 5 most sensitive types, and performed a 7-minute resting fMRI scan on them. Grouped by different trigger types, it turns out that the functional connectivity pattern of each group was unique, and the sensitivity to two trigger categories was positively correlated with the dorsal attention network, suggesting that ASMR may involve atypical attention processing.

\section{Relationship between ASMR and Other Similar Psychological Phenomena}

Whether it is the different personality traits, neural network activities, or other individual differences between ASMR experiencers and non-sense individuals, it reveals that ASMR may be some kind of basic difference related to cognition, feeling, and attention. In terms of the application of ASMR, some researchers pay more attention to the relationship between ASMR and some similar psy- 
chological phenomena in order to understand its causes and application methods.

del Campo and Kehle (2016) presented an argument that ASMR and frisson are interrelated in that they appear to arise through similar physiological mechanisms, and both may be induced or enhanced through the practice of mindfulness. More explicitly, ASMR might be regarded as a mild form of frisson. Kovacevich and Huron (2018) conducted a content analysis of 30 popular ASMR videos and 3600 comments. The results indicated that the physiological responses to ASMR (skin-related tingling and goosebumps) strongly resembled the classic frisson experience.

McErlean and Banissy (2018) believed that ASMR shares common characteristics with another underreported condition known as misophonia. Misophonia is a condition in which particular sounds elicit disproportionally strong aversive reactions (Rouw \& Erfanian, 2018). They compared the Misophonia Questionnaire (MQ) scores of ASMR experiencers and non-experiencers, Compared to controls, ASMR group scored higher on all subscales of MQ including the Misophonia Symptom Scale, the Misophonia' Emotions and Behaviors Scale and the Misophoriia Severity Scale. Individuals reporting ASMR experience have elevated levels of misophonia. Another large-scale online test found that ASMR and misophonia have the same characteristic of highly specific and individually different trigger-to-response associations (Rouw \& Erfanian, 2018). 385 participants finished this survey with 55 items, including yes/no questions, multiple answer questions, and open-ended questions, which collected information about their experience of ASMR and misophonia. Half of the participants reported their tingling sensations and unusual emotional responses to particular sound beyond their misophonia.

Fredborg et al. (2018) recruited 135 ASMR experiencers and 123 non-experiencers. They were asked to fill in the Totonto Mindfulness Scale (TMS) and the Mindful Attention and Awareness Scale (MAAS). Comparison of the two groups showed that individuals with ASMR generated significantly higher scores on the MAAS, a global measure of mindfulness, as well as significantly higher scores on the Curiosity subscale of the TMS. These results suggest that the sensory-emotional experiences associated with ASMR may be partially explained by a distinct subset of characteristics associated with mindfulness. Similarly, Barratt and Davis (2015) proposed that action of inducing ASMR may be a form of mindfulness, which intentionally focus one's attention to the internal and external experiences occurring in the present moment. Some researchers further investigated the differences in the flow, absorbtion and mindfulness of ASMR experienced and non-sensitized subjects (McErlean \& Osborne-Ford, 2020), and found that the two groups only differed in absorption. In the other two cases, the difference was not significant, indicating that the ASMR status may be associated with the ability to go deeply immersed with the current experience.

The similarity and high correlation between ASMR and frisson and misopho- 
nia allow us to guess the possible formation mechanism of ASMR from the perspective of those two. The high correlation with mindfulness reveals the potential for assisting psychotherapy. In future study, the ethical research and legalization of ASMR video can be a problem, because many ASMR videos contain sexual cues such as kiss sounds. People's perception of ASMR video is also polarized: people who can experience it find it useful, and others who have no similar experience feel bored what moderators affect the ASMR effect are waiting for investigating. Experimental studies of these two types of people may also reveal some of the mechanisms of ASMR.

\section{Conclusion}

There is still room for exploration in the application of ASMR in psychotherapy, especially in the fields of mindfulness, flow, and meditation. In daily life, ASMR already has a wide range of fans. Many video viewers aim at opportunities for relaxation and promotion of sleep, some others also report purposes of dealing with depression, anxiety and chronic pain (Barratt \& Davis, 2015; Rouw \& Erfanian, 2017). With the development of the Internet and technological innovation of video production, ASMR videos are much easier to achieve on contrast to the hardness of getting professional psychotherapy, thus a lot more people under pressure can benefit from it. Simply use a networked device with a pair of earphones and you can enjoy a rare personal relaxation at anywhere you like in 10 minutes. In addition, as the covid-19 spreads globally, many people are trapped at home, and negative emotions such as anxiety and depression increase. ASMR, as a potential intervention method mainly circulating on the Internet, maybe more feasible and can play a greater role than conventional psychological treatment programs.

\section{Conflicts of Interest}

The authors declare no conflicts of interest regarding the publication of this paper.

\section{References}

Barratt, E. L., \& Davis, N. J. (2015). Autonomous Sensory Meridian Response (ASMR): A Flow-Like Mental State. PeerJ, 3, Article ID: e851. https://doi.org/10.7717/peerj.851

Barratt, E. L., Spence, C., \& Davis, N. J. (2017). Sensory Determinants of the Autonomous Sensory Meridian Response (ASMR): Understanding the Triggers. Peer), 5, Article ID: e3846. https://doi.org/10.7717/peerj.3846

del Campo, M. A., \& Kehle, T. J. (2016). Autonomous Sensory Meridian Response (ASMR) and Frisson: Mindfully Induced Sensory Phenomena that Promote Happiness. International Journal of School \& Educational Psychology, 4, 99-105. https://doi.org/10.1080/21683603.2016.1130582

Fredborg, B. K., Clark, J. M., \& Smith, S. D. (2018). Mindfulness and Autonomous Sensory Meridian Response (ASMR). PeerJ, 6, Article ID: e5414.

https://doi.org/10.7717/peerj.5414 
Kovacevich, A., \& Huron, D. (2018). Two Studies of Autonomous Sensory Meridian Response (ASMR): The Relationship between ASMR and Music-Induced Frisson. Empirical Musicology Review, 13, 39-63. https://doi.org/10.18061/emr.v13i1-2.6012

McErlean, A. B. J., \& Banissy, M. J. (2017). Assessing Individual Variation in Personality and Empathy Traits in Self-Reported Autonomous Sensory Meridian Response. Multisensory Research, 30, 601-613. https://doi.org/10.1163/22134808-00002571

McErlean, A. B. J., \& Banissy, M. J. (2018). Increased Misophonia in Self-Reported Autonomous Sensory Meridian Response. Peer), 6, Article ID: e5351. https://doi.org/10.7717/peerj.5351

McErlean, A. B. J., \& Osborne-Ford, E. J. (2020). Increased Absorption in Autonomous Sensory Meridian Response. PeerJ, 8, Article ID: e8588.

https://doi.org/10.7717/peerj.8588

Roberts, N., Beath, A., \& Boag, S. (2020). A Mixed-Methods Examination of Autonomous Sensory Meridian Response: Comparison to Frisson. Consciousness and Cognition, 86, Article ID: 103046. https://doi.org/10.1016/j.concog.2020.103046

Rouw, R., \& Erfanian, M. (2017). A Large-Scale Study of Misophonia. Journal of Clinical Psychology, 74, 453-479. https://doi.org/10.1002/jclp.22500

Smith, S. D., Fredborg, B. K., \& Kornelsen, J. (2017). An Examination of the Default Mode Network in Individuals with Autonomous Sensory Meridian Response (ASMR). Social Neuroscience, 12, 361-365. https://doi.org/10.1080/17470919.2016.1188851

Smith, S. D., Fredborg, B. K., \& Kornelsen, J. (2019). Atypical Functional Connectivity Associated with Autonomous Sensory Meridian Response: An Examination of Five Resting-State Networks. Brain Connectivity, 9, 508-518.

https://doi.org/10.1089/brain.2018.0618

Smith, S. D., Fredborg, B. K., \& Kornelsen, J. (2020). Functional Connectivity Associated with Five Different Categories of Autonomous Sensory Meridian Response (ASMR) Triggers. Consciousness and Cognition, 85, Article ID: 103021.

https://doi.org/10.1016/j.concog.2020.103021 